\title{
RELIABILITY OF LINEAR SYSTEMS SUBJECTED TO WIND LOADS
}

\author{
A Thesis \\ Presented to the Faculty of the Graduate School \\ of Cornell University \\ in Partial Fulfillment of the Requirements for the Degree of \\ Master of Science
}

by

Haoran Zhao

August 2015 
(c) 2015 Haoran Zhao

ALL RIGHTS RESERVED 


\begin{abstract}
Probabilistic models for wind loads are developed and used to estimate the properties of the responses of linear systems. This analysis involves four steps. First, along-wind, across-wind forces and torque are represented as polynomials of turbulence fluctuations and wake excitations which are assumed to be stationary Gaussian processes. Second, we provide two types of models, namely, the empirical model and the mathematical model, for the second-moment properties of the turbulence fluctuations and wake excitations so that the probability law of the wind loads is characterized completely. Proposed models are then calibrated to the experimental observations. Third, the mathematical model provides an efficient method to estimate the response properties relative to Monte Carlo simulation. The responses are modeled by translation processes that match the target second-moment properties and marginal distributions of the responses. Fourth, the response properties which are of interest, e.g., the mean rates at which the responses exit the safe set, are calculated from the resulting translation processes. We illustrate this procedure by one numerical example.
\end{abstract}




\section{BIOGRAPHICAL SKETCH}

Born in $\mathrm{Xi}^{\prime}$ An, China, the author moved to Shanghai, China at his early age. He received the Bachelor of Engineering in Department of Civil Engineering at Tongji University. During his undergraduate study, he is also the caption of the student steel bridge competition in both ASCE Mid-Pacific and national student conferences held in Berkeley and Clemson, respectively. Then, he studied under the guidance of Professor Mircea Grigoriu in School of Civil and Enrionmental Engineering at Cornell University. His research includes construction of probabilistic modeles for both wind velocities and loads, reliability analysis of linear systems under wind environment, and evaluation of the performances of different decomposition techniques. 
This thesis is dedicated to my parents, Wei Zhao and Tong Zhu.

Without their love, support, and faith, this achievement would never have been possible. 


\section{ACKNOWLEDGEMENTS}

I am grateful for the support and assistance of many people who made the completion of this thesis possible. First, I wish to thank my advisor Professor Mircea Grigoriu. He continually and convincingly conveyed a spirit of adventure and provided invaluable expertise and theoretical insights in regard to my research.

Second, I would like to thank my committee members, Professor Huseyin Topaloglu, Professor Max Zhang, and Professor Peter Diamessis for their assistance and suggestions throughout the last three years.

Further thanks go to Dr. Emil Simiu, Professor luigi Carassale, Professor Yukio Tamura for the technical discussions regarding experimental datasets and numerical simulations and Dr. Alin Radu, Dr. Changhoon Lee for their help and encouragement.

Finally, I am grateful to my parents, who have been a constant source of inspiration and support throughout my studies and my life. 


\section{TABLE OF CONTENTS}

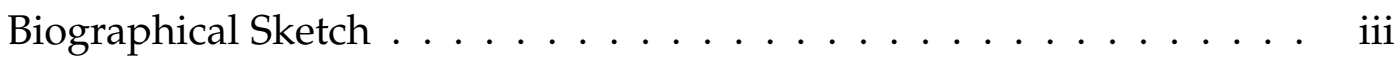

Dedication ................... iv

Acknowledgements ................... $\mathrm{v}$

Table of Contents . . . . . . . . . . . . . . . v vi

1 Introduction 1

2 Probabilistic models for wind loads 3

2.1 Wind loads on slender rigid building . . . . . . . . . . . . . 3

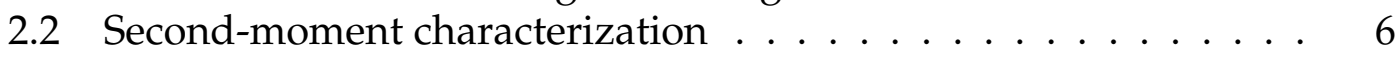

2.3 Turbulence and wake excitation modeling . . . . . . . . . . 7

3 Experimental data and model calibration 11

3.1 Experimental data . . . . . . . . . . . . . . . . . . 11

3.2 Model calibrations . . . . . . . . . . . . . . . . 12

$\begin{array}{llr}4 & \text { Response analysis } & 14\end{array}$

4.1 Second-moment properties ... . . . . . . . . . . . 14

4.2 Marginal moments and distributions . . . . . . . . . . . . . . . . . . . . 15

4.3 Response translation models . . . . . . . . . . . . . . 18

4.4 Mean outcrossing rates . . . . . . . . . . . . . . . . . . . . 19

5 Conclusions $\quad 21$

$\begin{array}{lr}\text { A Property from flow symmetry } & 22\end{array}$

B Proof of independence $\quad 23$

$\begin{array}{ll}\text { Bibliography } & 25\end{array}$ 


\section{CHAPTER 1}

\section{INTRODUCTION}

Experimental and mathematical approaches are used to characterize structural responses induced by wind loads. Experimental approaches include the fullscale and/or wind tunnel tests which provide valuable information on both wind loads on structures and structural responses. Cost limits this approach [1]. Mathematical approaches construct deterministic and probabilistic models for wind loads which can be used to estimate the properties of structural responses. We propose probabilistic models to represent wind loads, calibrate these models to the experimental observations, and assess the structural reliability for windload effects subjected to these models.

Our probabilistic models for wind loads are based on the wind-load model proposed in [7]. Three components of the wind loads, i.e., along-wind, acrosswind forces and torque, are defined by polynomials of the fluctuating part of the wind velocities. Because of the vortex wake formed at the rear region of the structure, additional forces are applied to capture the effects of the wake excitations. Then, the wind loads are fully described by the turbulence fluctuation and wake excitation terms.

Two types of the models for turbulence fluctuations and wake excitations are considered. The first model is the empirical model which has been used extensively in turbulence and wake excitation modeling. The functional forms of this model result from large amounts of experimental observations and satisfy the physics of the turbulence [8]. The second model represents the turbulence fluctuation and wake excitation terms by filtered Gaussian processes whose second-moment properties are similar to the empirical model, referred 
to as the mathematical model [5]. This model is used to develop an efficient method for calculating the response properties of linear structures. The method is based on translation processes which match the target marginal distributions as well as the second-moment properties of the responses. The first two moments of the responses are obtained by methods of the linear random vibration theory $[5,10]$ and marginal distributions are fitted to the marginal moments calculated exactly by Itô formula following an approach in [4]. Then, the structural reliability can be estimated from the resulting translation processes. We use the mean rate at which the structural responses exit the safe set, referred to as the mean outcrossing rate or mean failure rate, to characterize the structural reliability.

The outline of the thesis is as follows. The first section presents the probabilistic models for wind loads including the existing wind-load model and two models for turbulence fluctuations and wake excitations. The second section describes the experimental records we use and the algorithm of model calibration. The construction of the translation processes for responses, and the use of the method to approximate the mean outcrossing rate are in the third section. 


\section{CHAPTER 2}

\section{PROBABILISTIC MODELS FOR WIND LOADS}

The existing wind-load model is summarized first, then we use this model to characterize the second-moment properties of the wind loads and present two types of models for turbulence fluctuations and wake excitations.

\subsection{Wind loads on slender rigid building}

Consider a slender rigid building immersed in an ideal bi-dimensional wind field with wind velocity $V(\mathbf{x}, t)=[u(\mathbf{x}, t), v(\mathbf{x}, t)]$, where $\mathbf{x}=\left[x_{1}, x_{2}, x_{3}\right]$ is the spatial coordinates shown in Fig. 2.1(a), and $u(\mathbf{x}, t)$ and $v(\mathbf{x}, t)$ are the wind velocities along with and perpendicular to the mean wind direction $\beta$ (measured by the angle between $x_{1}$ and $x_{0,1}$, as shown in Fig. 2.1(b)), referred to as alongwind and across-wind velocities, respectively. Under the assumption that the wind field is stationary, the along-wind and across-wind velocities $u(\mathbf{x}, t)$ and $v(\mathbf{x}, t)$ admit the representations [8]

$$
u(\mathbf{x}, t)=\bar{U}(\mathbf{x})+\tilde{u}(\mathbf{x}, t), \text { and } v(\mathbf{x}, t)=\tilde{v}(\mathbf{x}, t),
$$

where $\bar{U}(\mathbf{x})$ is the mean wind velocity, and $\tilde{u}(\mathbf{x}, t)$ and $\tilde{v}(\mathbf{x}, t)$ are the along-wind and across-wind turbulence fluctuations. The building is idealized as a linear system with lumped masses at the floor levels. Each lumped mass has three degrees-of-freedom: along-wind, across-wind displacements and rotation. Let $\mathbf{F}(t)$ be the total force at one floor, and $F_{x}(t), F_{y}(t)$, and $M_{z}(t)$ be the alongwind, across-wind forces, and torque corresponding to the degrees-of-freedom, as shown in Fig. 2.1(a) and (b). 

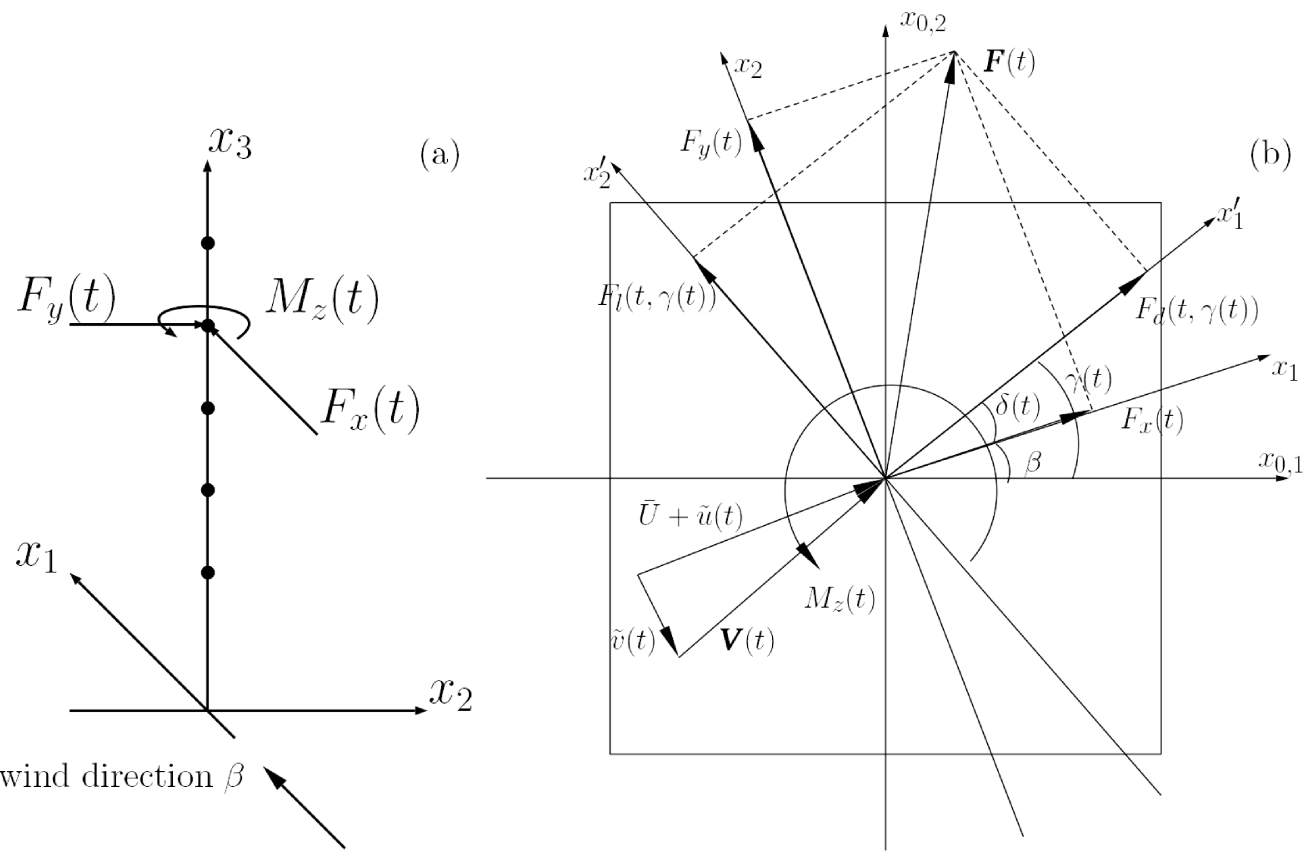

Mean wind direction $\beta$

Figure 2.1: Side (a) and top (b) views of the building

We adopt the wind-load model proposed in [7] to represent these forces. The construction of this model involves three steps. First, denote by $F_{d}(t), F_{l}(t)$ the projections of $\mathbf{F}(t)$ on the coordinate system aligned with the instantaneous direction of the wind velocity $\gamma(t)=\beta+\delta(t)$ (measured by the angle between $x_{1}^{\prime}$ and $\left.x_{0,1}\right)$, where $\delta(t)=\arcsin [\tilde{v}(t) / V(t)]$, in which $H_{3}$ is the height of the floor, $\bar{U}=\bar{U}\left(\left[0,0, H_{3}\right]\right), \tilde{u}(t)=\tilde{u}\left(\left[0,0, H_{3}\right], t\right), \tilde{v}(t)=\tilde{v}\left(\left[0,0, H_{3}\right], t\right)$, and $V(t)=$ $\sqrt{[\bar{U}+\tilde{u}(t)]^{2}+\tilde{v}^{2}(t)}$. As shown in Fig. 2.1(b), $F_{x}(t)$ and $F_{y}(t)$ relate $F_{d}(t)$ and $F_{l}(t)$ through equations

$$
\begin{aligned}
& F_{x}(t)=F_{d}(t) \cos \delta(t)-F_{l}(t) \sin \delta(t), \\
& F_{y}(t)=F_{d}(t) \sin \delta(t)+F_{l}(t) \cos \delta(t) .
\end{aligned}
$$

Second, set

$$
F_{d}(t)=\rho V^{2}(t) C_{d}(\gamma(t)) B / 2 \text {, and } F_{l}(t)=\rho V^{2}(t) C_{l}(\gamma(t)) B / 2,
$$

where $\rho$ is the air density, $B$ is a reference width of the building, and $C_{d}(\gamma(t))$ 
and $C_{l}(\gamma(t))$ are the non-dimensional force coefficients corresponding to $F_{d}(t)$ and $F_{l}(t)$, respectively [7]. The Taylor expansion of $C_{d}(\gamma(t))$ and $C_{l}(\gamma(t))$ at $\beta$ gives

$$
C_{d}(\gamma(t))=C_{d}+C_{d}^{\prime} \delta(t)+O\left(\delta^{2}(t)\right), \text { and } C_{l}(\gamma(t))=C_{l}+C_{l}^{\prime} \delta(t)+O\left(\delta^{2}(t)\right),
$$

where $C_{k}=C_{k}(\beta)$, and $C_{k}^{\prime}=\partial C_{k}(\gamma(t)) /\left.\partial \gamma(t)\right|_{\gamma(t)=\beta}, k=d, l$. We substitute Eqs. 2.4 and 2.5 into Eqs. 2.2 and 2.3. Under the assumption of small $\delta(t)$, Eqs. 2.2 and 2.3 become

$$
\begin{aligned}
& F_{x}(t)=\frac{1}{2} \rho \bar{U}^{2} B C_{d}+\rho \bar{U} \tilde{u}(t) B C_{d}+\frac{1}{2} \rho \tilde{u}^{2}(t) B C_{d}+\frac{1}{2} \rho \bar{U} \tilde{v}(t) B\left(C_{d}^{\prime}-C_{l}\right), \\
& F_{y}(t)=\frac{1}{2} \rho \bar{U}^{2} B C_{l}+\rho \bar{U} \tilde{u}(t) B C_{l}+\frac{1}{2} \rho \bar{U} \tilde{v}(t) B\left(C_{d}+C_{l}^{\prime}\right),
\end{aligned}
$$

Analogously, torque $M_{z}(t)$ has the form

$$
M_{z}(t)=\frac{1}{2} \rho \bar{U}^{2} B^{2} C_{m}+\rho \bar{U} \tilde{u}(t) B^{2} C_{m}+\frac{1}{2} \rho \bar{U} \tilde{v}(t) B^{2} C_{m}^{\prime},
$$

with the notations $C_{m}=C_{m}(\beta)$ and $C_{m}^{\prime}=\partial C_{m}(\gamma(t)) /\left.\partial \gamma(t)\right|_{\gamma(t)=\beta}$. Third, due to the presence of vortex shedding in the rear region of the building, additional forces associated with wake excitations are added linearly to Eqs. 2.6-2.8. Then, the non-dimensional forms of Eqs. 2.6-2.8 are

$$
\begin{aligned}
& C_{x}(t)=\frac{F_{x}(t)}{\frac{1}{2} \rho \bar{U}^{2} B}=C_{d}+2 \frac{\tilde{u}(t)}{\bar{U}} C_{d}+\frac{\tilde{u}^{2}(t)}{\bar{U}^{2}} C_{d}+\frac{\tilde{v}(t)}{\bar{U}}\left(C_{d}^{\prime}-C_{l}\right)+W_{x}(t), \\
& C_{y}(t)=\frac{F_{y}(t)}{\frac{1}{2} \rho \bar{U}^{2} B}=C_{l}+2 \frac{\tilde{u}(t)}{\bar{U}} C_{l}+\frac{\tilde{v}(t)}{\bar{U}}\left(C_{d}+C_{l}^{\prime}\right)+W_{y}(t), \text { and } \\
& C_{z}(t)=\frac{M_{z}(t)}{\frac{1}{2} \rho \bar{U}^{2} B^{2}}=C_{m}+2 \frac{\tilde{u}(t)}{\bar{U}} C_{m}+\frac{\tilde{v}(t)}{\bar{U}} C_{m}^{\prime}+W_{z}(t)
\end{aligned}
$$

where $C_{x}(t), C_{y}(t)$, and $C_{z}(t)$ are non-dimensional along-wind, across-wind, and torsional force coefficients, and $W_{x}(t), W_{y}(t)$, and $W_{z}(t)$ are zero-mean along-wind, across-wind, and torsional force coefficients associated with wake excitations, respectively. 
Note that (1) turbulence fluctuations $\tilde{u}(t)$ and $\tilde{v}(t)$ can be modeled by Gaussian processes [5]. Assume that $W_{k}(t), k=x, y, z$, are independent Gaussian processes, then $C_{y}(t)$ and $C_{z}(t)$ are Gaussian processes while $C_{x}(t)$ is a nonGaussian process which is consistent with the experimental observations (See next section); (2) Eqs. 2.9-2.11 can be simplified for zero mean wind direction, i.e., $\beta=0$. In this special case, turbulence fluctuations $\tilde{u}(t)$ and $\tilde{v}(t)$ are regarded to be independent because of the symmetry of the flow field which indicates $E[\tilde{u}(t) \tilde{v}(t)]=0$ and experimental observations (See Appendix 1). Moreover, $C_{d}^{\prime}=C_{l}=C_{m}=0$, and $W_{x}(t)$ contributes insignificantly to $C_{x}(t)$ [9]. Accordingly, Eqs. 2.9-2.11 yield

$$
\begin{aligned}
& C_{x}(t)=C_{d}+2 \frac{\tilde{u}(t)}{\bar{U}} C_{d}+\frac{\tilde{u}^{2}(t)}{\bar{U}^{2}} C_{d}, \\
& C_{y}(t)=\frac{\tilde{v}(t)}{\bar{U}}\left(C_{d}+C_{l}^{\prime}\right)+W_{y}(t), \text { and } \\
& C_{z}(t)=\frac{\tilde{v}(t)}{\bar{U}} C_{m}^{\prime}+W_{z}(t) .
\end{aligned}
$$

In this special case, along-wind force coefficient $C_{x}(t)$ is independent of $C_{y}(t)$ and $C_{z}(t)$.

\subsection{Second-moment characterization}

Let $C(t)=\left[C_{x}(t), C_{y}(t), C_{z}(t)\right]^{T}$ be the non-dimensional wind-load vector process. We show the second-moment properties of $C(t)$ for the zero-mean-winddirection case. First, the mean of $C(t), E[C(t)]=\left[C_{d}\left(1+E\left[\tilde{u}^{2}(t)\right] / \bar{U}^{2}\right), 0,0\right]^{T}$. Second, denote by $\Gamma_{k q}(\tau)=E\left[C_{k}(t+\tau) C_{q}(t)\right]-E\left[C_{k}(t+\tau)\right] E\left[C_{q}(t)\right], k, q=x, y, z$, the covariance functions between two arbitrary components of $C(t)$, where $\tau$ denotes the time lag. The spectral densities of wind loads are $S_{k q}(\nu)=\mathcal{F}\left[\Gamma_{k q}(\tau)\right]$, where $\nu$ is the circular frequency and $\mathcal{F}$ is the Fourier Transform operator. These 
spectral densities have the expressions

$$
\begin{aligned}
& S_{x x}(\nu)=\frac{4 C_{d}^{2} S_{\tilde{u}}(\nu)}{\bar{U}^{2}}+\frac{C_{d}^{2} S_{\tilde{u}^{2}}(\nu)}{\bar{U}^{4}} \\
& S_{y y}(\nu)=\frac{\left(C_{d}+C_{l}^{\prime}\right)^{2} S_{\tilde{v}}(\nu)}{\bar{U}^{2}}+S_{W_{y}}(\nu) \\
& S_{z z}(\nu)=\frac{C_{m}^{\prime 2} S_{\tilde{v}}(\nu)}{\bar{U}^{2}}+S_{W_{z}}(\nu) \\
& S_{x y}(\nu)=S_{x z}(\nu)=0, \text { and } \\
& S_{y z}(\nu)=\frac{\left(C_{d}+C_{l}^{\prime}\right) C_{m}^{\prime} S_{\tilde{v}}(\nu)}{\bar{U}^{2}}
\end{aligned}
$$

with the notations $S_{\tilde{\eta}}(\nu)=\mathcal{F}\{E[\tilde{\eta}(t+\tau) \tilde{\eta}(t)]\}, \quad \eta=u, v, \quad S_{\tilde{u}^{2}}(\nu)=$ $\mathcal{F}\left\{E\left[\tilde{u}^{2}(t+\tau) \tilde{u}^{2}(t)\right]\right\}$, and $S_{W_{\xi}}(\nu)=\mathcal{F}\left\{E\left[W_{\xi}(t+\tau) W_{\xi}(t)\right]\right\}, \xi=y, z$. Eqs. 2.152.19 relate the wind-load spectral densities with the spectral densities of turbulence fluctuations and wake excitations for which we provide models in the following section.

\subsection{Turbulence and wake excitation modeling}

Two models for $S_{\tilde{\eta}}(\nu), \eta=u, v$, and $S_{W_{\xi}}(\nu), \xi=y, z$, are discussed. Model 1 is referred to as the empirical model which follows from an extensive series of experiment observations and some information regarding the physics of the turbulence. Model 2 represents the turbulence fluctuation and wake excitation terms by filtered Gaussian processes of which the functional forms are selected such that the resulting spectral densities match the empirical model as close as possible.

\section{Model 1: Empirical model}

The generalized empirical model for $S_{\tilde{\eta}}(\nu)$, denoted by $S_{\tilde{\eta}}^{(1)}(\nu)$, has the expres- 
sion [8]

$$
\frac{\nu S_{\tilde{\eta}}^{(1)}(\nu)}{U_{*}^{2}}=\frac{n S_{\tilde{\eta}}^{(1)}(n)}{U_{*}^{2}}=\frac{A_{\eta} f^{a_{\eta}}}{\left(C_{\eta}+B_{\eta} f^{b_{\eta}}\right)^{D_{\eta}}},
$$

where $n$ is the frequency measured in $\mathrm{Hz}, U_{*}$ is the friction velocity, $f=n H_{3} / \bar{U}$ is the Monin coordinates, and $A_{\eta}, B_{\eta}, C_{\eta}, D_{\eta}, a_{\eta}, b_{\eta}$ are the parameters to be determined. Denote by $f_{m, \eta}$ the $f$ value in correspondence of the peak value of $n S_{\tilde{\eta}}^{(1)}(n)$. Since the spectral densities in the inertial subrange are governed by the Kolmogorov hypotheses, i.e., $n S_{\tilde{\eta}}^{(1)}(n) / U_{*}^{2} \propto f^{-2 / 3}$, Eq. 2.20 can be simplified to

$$
\frac{\nu S_{\tilde{\eta}}^{(1)}(\nu)}{\sigma_{\eta}^{2}}=\frac{n S_{\tilde{\eta}}^{(1)}(n)}{\sigma_{\eta}^{2}}=\frac{c_{\eta} f / f_{m \eta} \gamma_{\eta}}{\left[1+1.5 \gamma_{\eta}\left(f / f_{m \eta}\right)^{\beta_{\eta}}\right]^{\frac{2 / 3+\gamma_{\eta}}{\beta_{\eta}}}},
$$

where $\sigma_{\eta}$ is the standard deviation of the turbulence, $\beta_{\eta}, \gamma_{\eta}$ are parameters defining the peakedness of the spectral densities, and $c_{\eta}=1 / \int_{0}^{\infty}\left[f / f_{m \eta}^{\gamma_{\eta}}\right] /[\nu+$ $\left.1.5 \nu \gamma_{\eta}\left(f / f_{m \eta}\right)^{\beta_{\eta}}\right]^{\frac{2 / 3+\gamma_{\eta}}{\beta_{\eta}}} d \nu$ guarantees that $\int_{0}^{\infty} S_{\tilde{\eta}}^{(1)}(\nu) d \nu=\sigma_{\eta}^{2}$.

The spectral density $S_{W_{\xi}}^{(1)}(\nu)$ based on the empirical model takes the form

$$
S_{W_{\xi}}^{(1)}(\nu)=\sigma_{W_{\xi}}^{2} g_{W_{\xi}}
$$

where $\sigma_{W_{\xi}}$ is the standard deviation of the force coefficients due to wake excitations and $g_{W_{\xi}}$ is assumed to have the form [11]

$$
g_{W_{\xi}}=\frac{1}{\sqrt{\pi} d_{\xi} \nu_{W_{\xi}}} \exp \left\{-\left[\frac{1-\frac{\nu}{\nu_{W_{\xi}}}}{d_{\xi}}\right]^{2}\right\}
$$

where $d_{\xi}$ is the bandwidth parameter and $\nu_{W_{\xi}}$ denotes the vortex shedding frequency.

Eqs. 2.21 and 2.22 generally provide satisfactory agreements with experimental observations (See next section). However, the empirical model has one limitation. Few methods can be utilized to estimate the properties of the responses. Classical Monte Carlo simulation is one of the candidate methods 
which infers the statistics of the responses from response samples that are obtained by solving deterministic versions of equation of motion corresponding to samples of $\tilde{u}(t), \tilde{v}(t), W_{y}(t)$, and $W_{z}(t)$. The required sample size is large if we analyze, e.g., mean outcrossing rate, so that the computational time can be significant.

\section{Model 2: Mathematical model}

We model the turbulence fluctuation and wake excitation terms by filtered Gaussian processes which have the similar spectral densities as the empirical model. Turbulence fluctuations $\tilde{u}(t)$ and $\tilde{v}(t)$ can be described by the OrnsteinUhlenbeck processes [5]

$$
d \tilde{\eta}(t)=-\alpha_{\eta} \tilde{\eta}(t) d t+\sigma_{\eta}^{2} \sqrt{2 \alpha_{\eta}} d B_{\eta}(t)
$$

and $W_{y}(t), W_{z}(t)$ are modeled by the responses of linear systems driven by Gaussian noise, i.e.,

$$
d \mathbf{W}_{\xi}(t)=\mathbf{D}_{\xi} \mathbf{W}_{\xi}(t) d t+\mathbf{g}_{\xi} d B_{\xi}(t)
$$

where $\mathbf{W}_{\xi}(t)=\left[W_{\xi}(t), \dot{W}_{\xi}(t)\right]^{T}, \mathbf{g}_{\xi}=\left[0, \beta_{\xi}\right]^{T}, \mathbf{D}_{\xi}$ is a matrix with components $\mathbf{D}_{\xi}(1,1)=0, \mathbf{D}_{\xi}(1,2)=1, \mathbf{D}_{\xi}(2,1)=-\theta_{\xi}^{2}, \mathbf{D}_{\xi}(2,2)=-2 \gamma_{\xi} \theta_{\xi}$, and $\left\{B_{\eta}(t), B_{\xi}(t)\right\}$ are independent standard Brownian motions. The spectral densities corresponding to the models in Eqs. 2.24 and 2.25 are

$$
\begin{aligned}
& S_{\tilde{\eta}}^{(2)}(\nu)=\mathcal{F}\{E[\tilde{\eta}(t) \tilde{\eta}(t+\tau)]\}=\frac{2 \alpha_{\eta} \sigma_{\eta}^{2}}{\left[\pi\left(\nu^{2}+\alpha_{\eta}^{2}\right)\right]}, \\
& S_{\tilde{u}^{2}}^{(2)}(\nu)=\mathcal{F}\left\{E\left[\tilde{u}^{2}(t) \tilde{u}^{2}(t+\tau)\right]\right\}=\frac{8 \alpha_{u} \sigma_{u}^{4}}{\left[\pi\left(\nu^{2}+4 \alpha_{u}^{2}\right)\right]}, \text { and } \\
& S_{W_{\xi}}^{(2)}(\nu)=\mathcal{F}\left\{E\left[W_{\xi}(t) W_{\xi}(t+\tau)\right]\right\}=\frac{\beta_{\xi}^{2}}{\left[\left(\nu^{2}-\theta_{\xi}^{2}\right)^{2}+4 \gamma_{\xi}^{2} \nu^{2} \theta_{\xi}^{2}\right]}
\end{aligned}
$$

where The parameters $\alpha_{\eta}, \sigma_{\eta}, \theta_{\xi}, \gamma_{\xi}, \beta_{\xi}$ are selected by minimizing the norms $\left\|S_{\tilde{\eta}}^{(2)}(\nu)-S_{\tilde{\eta}}^{(1)}(\nu)\right\|$ and $\left\|S_{W_{\xi}}^{(2)}(\nu)-S_{W_{\xi}}^{(1)}(\nu)\right\|$. Unlike the unfavorable feature of 
model 1, model 2 provides an alternative method to estimate the response properties. This method is more efficient than Monte Carlo simulation.

The structural responses are approximated by translation processes which require knowledge of the marginal distributions as well as the second-moment properties of the responses. The second-moment properties, i.e., the mean and correlation functions of responses, are calculated from those of the wind loads. Marginal distributions are fitted to the marginal moments which can be obtained from moment equations derived from the Itô's formula. The resulting translation processes can then be utilized to assess the structural performances. 


\section{CHAPTER 3}

\section{EXPERIMENTAL DATA AND MODEL CALIBRATION}

The experimental records we use in our analysis are described, several assumptions are validated, and the algorithm for model calibration is illustrated by one example.

\subsection{Experimental data}

The wind tunnel records from Shimizu Corporation Laboratories for zero wind direction are used in our analysis. The mean wind velocity profile corresponds to the urban terrain. The model is a 25-story building with square cross-section and has 500 wind pressure taps uniformly distributed on its sides. The length scale used for the model is 1:400. 32768 measurements of non-dimensional pressure coefficients are recorded continuously with $1000 \mathrm{~Hz}$ measurement frequency. Further details can be found in [6].

Under the assumption that the pressure field is stationary and ergodic, statistics of non-dimensional wind loads $C_{x}(t), C_{y}(t)$, and $C_{z}(t)$ can be estimated by averaging the time records. Table 1 shows the first four marginal moments of the wind loads at the $12^{\text {th }}$ floor. Denote by $\mu_{k}$ and $\sigma_{k}$ the mean and standard deviation of $C_{k}(t)$, and set $\tilde{C}_{k}(t)=\left[C_{k}(t)-\mu_{k}\right] / \sigma_{k}$ the normalized wind load. Fig. 3.1(a) shows the marginal densities of $\tilde{C}_{k}(t)$ and standard normal distribution. We can see that $\tilde{C}_{x}(t)$ presents more non-Gaussian features than $\tilde{C}_{y}(t)$ and $\tilde{C}_{z}(t)$. This observation supports the formulation of our wind-load model. Fig. 3.1(b) shows the correlation functions $E\left[\tilde{C}_{x}(t+\tau) \tilde{C}_{y}(t)\right]$, 
$E\left[\tilde{C}_{x}(t+\tau) \tilde{C}_{z}(t)\right]$, and $E\left[\tilde{C}_{y}(t+\tau) \tilde{C}_{z}(t)\right]$ which can be viewed as the corresponding correlation-coefficient functions between $\left(C_{x}(t), C_{y}(t)\right),\left(C_{x}(t), C_{z}(t)\right)$, and $\left(C_{y}(t), C_{z}(t)\right)$. It can be seen that $C_{x}(t)$ is almost uncorrelated with $C_{y}(t)$ and $C_{z}(t)$, i.e., $E\left[C_{x}(t+\tau) C_{y}(t)\right]=E\left[C_{x}(t+\tau) C_{z}(t)\right]=0$. From Eqs. 2.12-2.14, $E\left[C_{x}(t+\tau) C_{y}(t)\right]$ and $E\left[C_{x}(t+\tau) C_{z}(t)\right]$ are proportional to $E[\tilde{u}(t+\tau) \tilde{v}(t)]$. This implies the independence between $\tilde{u}(t)$ and $\tilde{v}(t)$ since we assume that $\tilde{u}(t)$ and $\tilde{v}(t)$ are Gaussian.

\begin{tabular}{|c|c|c|c|c|}
\hline Wind load & Mean & Variance & Skewness & Kurtosis \\
\hline$C_{x}(t)$ & 1.1250 & 0.0920 & 0.6305 & 3.7304 \\
$C_{y}(t)$ & -0.0322 & 0.2148 & -0.0262 & 3.0330 \\
$C_{z}(t)$ & -0.0083 & 0.0051 & 0.0272 & 3.5969 \\
\hline
\end{tabular}

Table 3.1: Statistics of non-dimensional wind loads
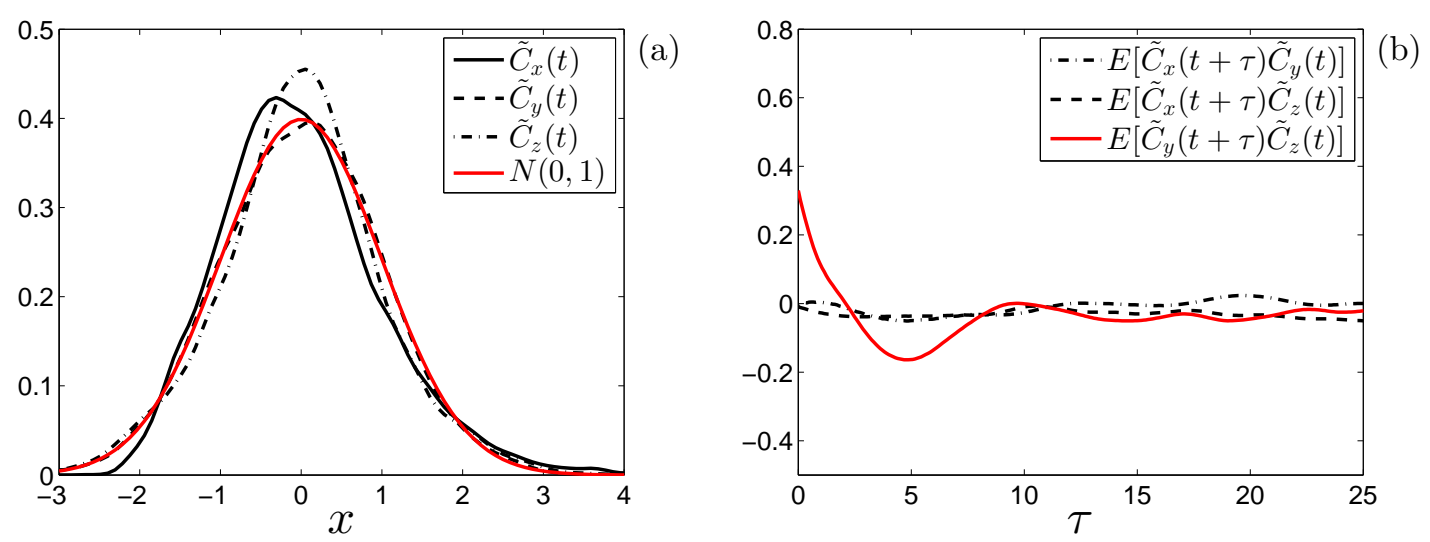

Figure 3.1: Statistics of $\tilde{C}_{x}(t), \tilde{C}_{y}(t)$, and $\tilde{C}_{z}(t)$ : Marginal densities (a) and Correlation functions (b)

\subsection{Model calibrations}

The parameters of both models defined are fitted to the experimental observations. Let $S_{k k}(\nu ; \Theta), k=x, y, z$, be the functional forms of spectral densities 
with a vector $\Theta$ of parameters, e.g., $\Theta=\left[\alpha_{u} \sigma_{u}\right]$ for the spectral density of the along-wind force based on model 2. The algorithm for model calibration has two steps. First, spectral densities of the wind loads are estimated from experimental records. Denote by $\hat{S}_{k k}(\nu), k=x, y, z$, the corresponding estimates of the spectral densities. Second, parameters $\Theta$ are selected by minimizing the error $\int\left[S_{k k}(\nu ; \Theta)-\hat{S}_{k k}(\nu)\right]^{2} d \nu$.

Fig. 3.2 shows one example for model calibration. The red solid lines are the estimates $\hat{S}_{k k}(\nu)$ obtained from experimental records. The blue dash and black solid lines are the spectral densities $S_{k k}(\nu ; \Theta)$ based on model 1 and 2 fitted to $\hat{S}_{k k}(\nu)$ by the above algorithm. We can see that both models match satisfactorily with the experimental observations.
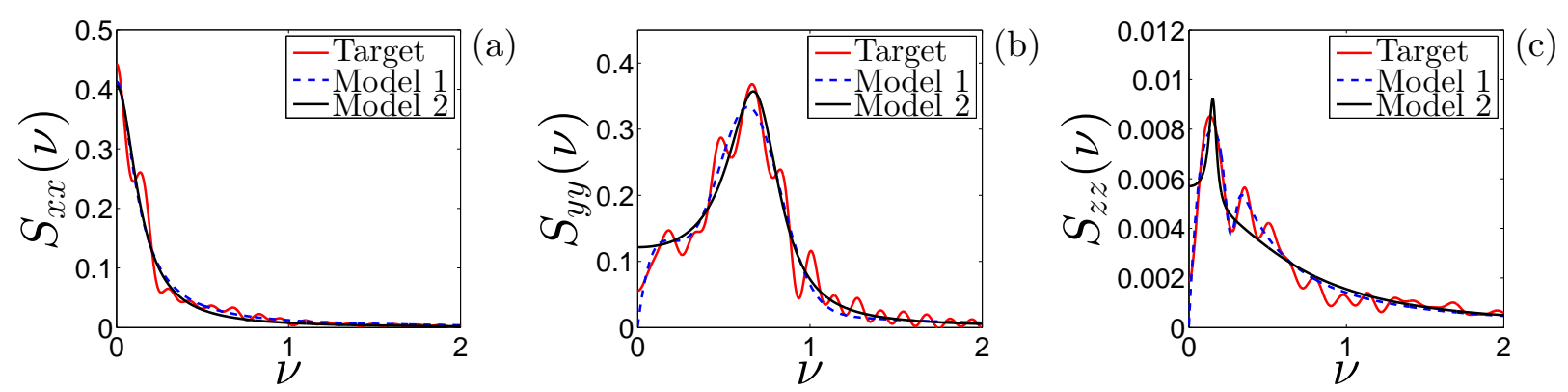

Figure 3.2: Example of model calibration for: along-wind force $C_{x}(t)(\mathrm{a})$, across-wind force $C_{y}(t)(\mathrm{b})$, and torque $C_{z}(t)(\mathrm{c})$ 


\section{CHAPTER 4}

\section{RESPONSE ANALYSIS}

We show the detailed procedure to construct translation models for the responses based on model 2. Let $\mathbf{X}(t)=\left[X_{1}(t), X_{2}(t), X_{3}(t)\right]^{T}$ be the 3-dimensional response vector process for the linear system. The components of $\mathbf{X}(t)$, $X_{1}(t), X_{2}(t)$, and $X_{3}(t)$, are along-wind, across-wind displacements and rotation, respectively. The vector process $\mathbf{X}(t)$ satisfies the equation of motion for a 3 degrees-of-freedom system

$$
\mathbf{M} \ddot{\mathbf{X}}(t)+\mathbf{D} \dot{\mathbf{X}}(t)+\mathbf{K X}(t)=\mathbf{C}(t)
$$

where $\mathbf{M}, \mathbf{D}$, and $\mathbf{K}$ are the mass, damping, and stiffness matrices and $\mathbf{C}(t)=$ $\left[C_{x}(t), C_{y}(t), C_{z}(t)\right]^{T}$ is the wind-load vector process, where $C_{x}(t), C_{y}(t)$, and $C_{z}(t)$ are given by Eqs. 2.12-2.14. Let $\boldsymbol{\Psi}=\left[\psi_{1}, \psi_{2}, \psi_{3}\right]$ be a $3 \times 3$ matrix whose columns are modal shapes and

$$
\mathbf{Y}(t)=\mathbf{\Psi}^{-1} \mathbf{X}(t)
$$

be the modal responses. Write Eq. 4.1 in terms of $\mathbf{Y}(t)$, we have

$$
\ddot{Y}_{k}(t)+2 \zeta_{k} \omega_{k} \dot{Y}_{k}(t)+\omega_{k}^{2} Y_{k}(t)=\frac{\hat{C}_{k}(t)}{M_{k}}, \quad k=1,2,3,
$$

where $Y_{k}(t)$ are the components of $\mathbf{Y}(t)$, and $\zeta_{k}, \omega_{k}$, and $M_{k}$ are modal damping ratios, natural frequencies, and masses. The modal forcing functions have the expressions $\hat{C}_{k}(t)=\left[\boldsymbol{\Psi}^{T} \mathbf{C}(t)\right]_{k}$. Without loss of generality, we set $M_{k}=1$.

\subsection{Second-moment properties}

The mean of $\mathbf{X}(t)$ is defined by $E[\mathbf{X}(t)]=\sum_{k=1}^{3} \psi_{k} E\left[Y_{k}(t)\right]=\sum_{k=1}^{3} \psi_{k} E\left[\hat{C}_{k}(t)\right] / \omega_{k}^{2}$. This equation results from Eqs. 4.2 and 4.3. 
Similarly, the spectral density of $\mathbf{X}(t)$, denoted by $\mathbf{S}_{\mathbf{X}}(\nu)$, is

$$
\mathbf{S}_{\mathbf{X}}(\nu)=\mathcal{F}\left\{E\left[\mathbf{X}(t+\tau) \mathbf{X}^{T}(t)\right]\right\}=\sum_{k=1}^{3} \sum_{q=1}^{3} \psi_{k} \psi_{q}^{T} S_{Y_{k} Y_{q}}(\nu),
$$

where $S_{Y_{k} Y_{q}}(\nu)$ is the cross spectral density of arbitrary modal displacement pairs $\left\{Y_{k}(t), Y_{q}(t)\right\}, k, q=1,2,3 . S_{Y_{k} Y_{q}}(\nu)$ can be calculated in two steps. First, $\left\{Y_{k}(t), Y_{q}(t)\right\}$ follows the equation [10, p. 189]

$$
\frac{d}{d t} \tilde{\mathbf{Y}}(t)=\mathbf{A} \tilde{\mathbf{Y}}(t)+\mathbf{g} \hat{\mathbf{C}}(t)=\left[\begin{array}{cc}
\mathbf{A}_{k} & \mathbf{0} \\
\mathbf{0} & \mathbf{A}_{q}
\end{array}\right] \tilde{\mathbf{Y}}(t)+\mathbf{g} \hat{\mathbf{C}}(t)
$$

where $\tilde{\mathbf{Y}}(t)=\left[Y_{k}(t), \dot{Y}_{k}(t), Y_{q}(t), \dot{Y}_{q}(t)\right]^{T}, \hat{\mathbf{C}}(t)=\left[\hat{C}_{k}(t), \hat{C}_{q}(t)\right]^{T}, \mathbf{g}$ is a $4 \times 2$ matrix with the first column $[0,1,0,0]^{T}$ and the second column $[0,0,0,1]^{T}$, and $\mathbf{A}_{k}$ is a $2 \times 2$ matrix with components $\mathbf{A}_{k}(1,1)=0, \mathbf{A}_{k}(1,2)=1, \mathbf{A}_{k}(2,1)=-\omega_{k}^{2}$, and $\mathbf{A}_{k}(2,2)=-2 \zeta_{k} \omega_{k}$. Second, let $\mathbf{S}_{\tilde{\mathbf{Y}}}(\nu)=\mathcal{F}\left\{E\left[\tilde{\mathbf{Y}}(t+\tau) \tilde{\mathbf{Y}}^{T}(t)\right]\right\}$ and $\mathbf{S}_{\hat{\mathbf{C}}}(\nu)=$ $\mathcal{F}\left\{E\left[\hat{\mathbf{C}}(t+\tau) \hat{\mathbf{C}}^{T}(t)\right]\right\}$ be the spectral densities of $\tilde{\mathbf{Y}}(t)$ and $\hat{\mathbf{C}}(t)$, respectively. $\mathbf{S}_{\tilde{\mathbf{Y}}}(\nu)$ relates $\mathbf{S}_{\hat{\mathbf{C}}}(\nu)$ through the equation [10, p. 195]

$$
\mathbf{S}_{\tilde{\mathbf{Y}}}(\nu)=\mathbf{H}^{*}(\nu) \mathbf{S}_{\hat{\mathbf{C}}}(\nu) \mathbf{H}^{T}(\nu),
$$

where $*$ indicates the complex conjugate and $\mathbf{H}(\nu)=(\sqrt{-1} \nu \mathbf{I}-\mathbf{A})^{-1} \mathbf{g}$, in which I is a $4 \times 4$ identity matrix. $S_{Y_{k} Y_{q}}(\nu)$ is the component $(1,3)$ or $(3,1)$ of $\mathbf{S}_{\tilde{\mathbf{Y}}}(\nu)$.

Fig. 4.1 shows one example for the spectral densities of modal responses. The solid line is the estimate from experimental records and dash line is calculated from Eq. 4.6.

\subsection{Marginal moments and distributions}

We use moment equations developed from Itô's formula to calculate exactly the marginal moments of $\mathbf{X}(t)$, then infer its marginal distributions. 

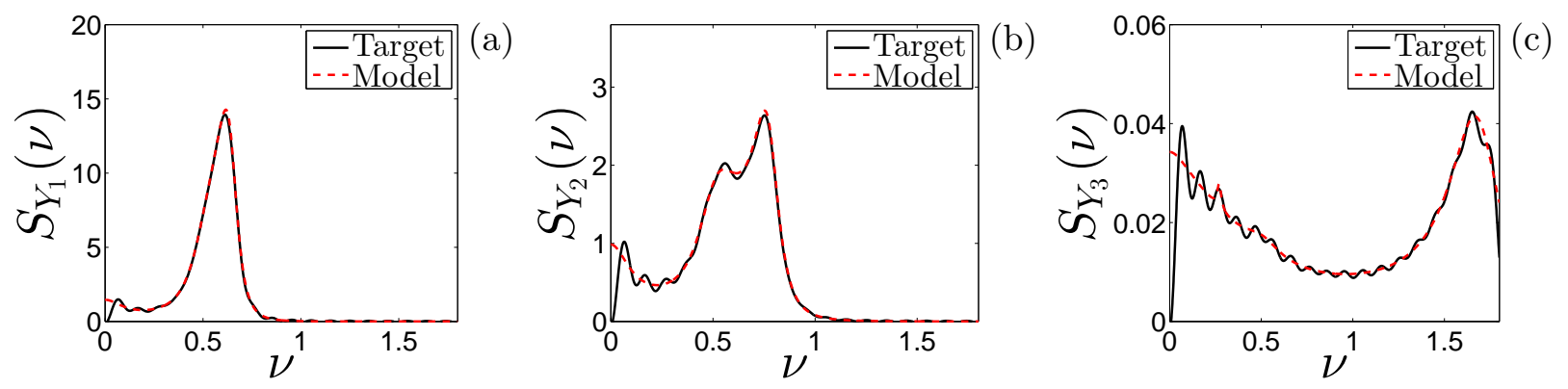

Figure 4.1: Example for the spectral densities of modal responses: $Y_{1}(t)(\mathrm{a})$, $Y_{2}(t)(\mathrm{b})$, and $Y_{3}(t)(\mathrm{c})$

Let $X_{i}(t), i=1,2,3$, be the $i^{t h}$ component of $\mathbf{X}(t)$. The $n^{\text {th }}$ marginal moments $E\left[X_{i}^{n}(t)\right]$ of $X_{i}(t)$ has the expression

$$
\begin{aligned}
E\left[X_{i}^{n}(t)\right] & =E\left[\left(\psi_{i, 1} Y_{1}(t)+\psi_{i, 2} Y_{2}(t)+\psi_{i, 3} Y_{3}(t)\right)^{n}\right] \\
& =\sum_{\alpha, \beta, \gamma} \frac{n !}{\alpha ! \beta ! \gamma !} \psi_{i, 1}^{\alpha} \psi_{i, 2}^{\beta} \psi_{i, 3}^{\gamma} E\left[Y_{1}^{\alpha}(t) Y_{2}^{\beta}(t) Y_{3}^{\gamma}(t)\right]
\end{aligned}
$$

in which $\alpha+\beta+\gamma=n$, and $\psi_{i, 1}, \psi_{i, 2}$, and $\psi_{i, 3}$ are the first, second, and third component of mode $\psi_{i}$, respectively. Note that $Y_{k}(t), k=1,2,3$, are governed by Eq. 4.3 and the $\hat{C}_{k}(t)$ term in Eq. 4.3 has the expression $\hat{C}_{k}(t)=\psi_{k, 1} C_{x}(t)+$ $\psi_{k, 2} C_{y}(t)+\psi_{k, 3} C_{z}(t)$. Substituting the above expression in Eq. 4.3 gives

$$
\ddot{Y}_{k}(t)+2 \zeta_{k} \omega_{k} \dot{Y}_{k}(t)+\omega_{k}^{2} Y_{k}(t)=\psi_{k, 1} C_{x}(t)+\psi_{k, 2} C_{y}(t)+\psi_{k, 3} C_{z}(t)
$$

Since Eq. 4.8 is linear, we decompose $Y_{k}(t)$ by $Y_{k}(t)=\psi_{k, 1} Y_{k, x}(t)+\psi_{k, 2} Y_{k, y}(t)+$ $\psi_{k, 3} Y_{k, z}(t)$, where $Y_{k, x}(t), Y_{k, y}(t)$, and $Y_{k, z}(t)$ are the responses corresponding to wind loads $C_{x}(t), C_{y}(t)$, and $C_{z}(t)$, respectively. Then, $E\left[Y_{1}(t)^{\alpha} Y_{2}(t)^{\beta} Y_{3}(t)^{\gamma}\right]$ are calculated from the moments of $Y_{k, x}(t), Y_{k, y}(t)$ and $Y_{k, z}(t)$ by

$$
\begin{gathered}
E\left[Y_{1}^{\alpha}(t) Y_{2}^{\beta}(t) Y_{3}^{\gamma}(t)\right]=\sum_{\alpha_{1}, \alpha_{2}, \alpha_{3}} \sum_{\beta_{1}, \beta_{2}, \beta_{3}} \sum_{\gamma_{1}, \gamma_{2}, \gamma_{3}} \frac{\alpha !}{\alpha_{1} ! \alpha_{2} ! \alpha_{3} !} \frac{\beta !}{\beta_{1} ! \beta_{2} ! \beta_{3} !} \frac{\gamma !}{\gamma_{1} ! \gamma_{2} ! \gamma_{3} !} \prod_{i=1}^{3} \psi_{1, i}^{\alpha_{i}} \prod_{j=1}^{3} \psi_{2, j}^{\beta_{j}} \prod_{k=1}^{3} \psi_{3, k}^{\gamma_{k}} \\
E\left[Y_{1, x}^{\alpha_{1}}(t) Y_{2, x}^{\beta_{1}}(t) Y_{3, x}^{\gamma_{1}}(t)\right] E\left[Y_{1, y}^{\alpha_{2}}(t) Y_{2, y}^{\beta_{2}}(t) Y_{3, y}^{\gamma_{2}}(t) Y_{1, z}^{\alpha_{3}}(t) Y_{2, z}^{\beta_{3}}(t) Y_{3, z}^{\gamma_{3}}(t)\right]
\end{gathered}
$$


where $\alpha_{1}+\alpha_{2}+\alpha_{3}=\alpha, \beta_{1}+\beta_{2}+\beta_{3}=\beta$, and $\gamma_{1}+\gamma_{2}+\gamma_{3}=$ $\gamma$. The above equation follows from the fact that $Y_{k, x}(t)$ are independent of $Y_{k, y}(t)$ and $Y_{k, z}(t)$. Since $C_{y}(t)$ and $C_{z}(t)$ are correlated Gaussian processes so are the responses $Y_{k, y}(t)$ and $Y_{k, z}(t)$, then higher order moments of $Y_{k, y}(t)$ and $Y_{k, z}(t)$ in Eq. 4.9 result from their second-moment properties. Let $\mu\left(\alpha_{1}, \alpha_{1}^{\prime}, \alpha_{2}, \alpha_{2}^{\prime}, \alpha_{3}, \alpha_{3}^{\prime}, r\right)=E\left[Y_{1, x}^{\alpha_{1}}(t) \dot{Y}_{1, x}^{\alpha_{1}^{\prime}}(t) Y_{2, x}^{\beta_{1}}(t) \dot{Y}_{2, x}^{\beta_{1}^{\prime}}(t) Y_{3, x}^{\gamma_{1}}(t) \dot{Y}_{3, x}^{\gamma_{1}^{\prime}}(t) \tilde{u}^{r}(t)\right]$ be the moments of vector $\left[Y_{1, x}(t), \dot{Y}_{1, x}(t), Y_{2, x}(t), \dot{Y}_{2, x}(t), Y_{3, x}(t), \dot{Y}_{3, x}(t), \tilde{u}(t)\right]$. Note that $\mu\left(\alpha_{1}, \alpha_{1}^{\prime}, \alpha_{2}, \alpha_{2}^{\prime}, \alpha_{3}, \alpha_{3}^{\prime}, r\right)$ satisfy the moment equations derived from the Itô's formula [4], i.e.,

$$
\begin{aligned}
0= & \alpha_{1} \mu\left(\alpha_{1}-1, \alpha_{1}^{\prime}+1, \ldots\right)+\alpha_{2} \mu\left(\ldots, \alpha_{2}-1, \alpha_{2}^{\prime}+1, \ldots\right)+\alpha_{3} \mu\left(\ldots, \alpha_{3}-1, \alpha_{3}^{\prime}+1, r\right) \\
& -\alpha_{1}^{\prime} \omega_{1}^{2} \mu\left(\alpha_{1}+1, \alpha_{1}^{\prime}-1, \ldots\right)-\beta_{1}^{\prime} \omega_{2}^{2} \mu\left(\ldots, \beta_{1}+1, \beta_{1}^{\prime}-1, \ldots\right)-\gamma_{1}^{\prime} \omega_{3}^{2} \mu\left(\ldots, \gamma_{1}+1, \gamma_{1}^{\prime}-1, r\right) \\
& -\left(2 \alpha_{1}^{\prime} \zeta_{1} \omega_{1}+2 \beta_{1}^{\prime} \zeta_{2} \omega_{2}+2 \gamma_{1}^{\prime} \zeta_{3} \omega_{3}+r \alpha_{u}\right) \mu(\ldots)+\alpha_{1}^{\prime} C_{d} \sum_{p=0}^{2} a_{l} \mu\left(\alpha_{1}, \alpha_{1}^{\prime}-1, \ldots, r+l\right) \\
& +\beta_{1}^{\prime} C_{d} \sum_{p=0}^{2} a_{l} \mu\left(\ldots, \beta_{1}, \beta_{1}^{\prime}-1, \ldots, r+l\right)+\gamma_{1}^{\prime} C_{d} \sum_{p=0}^{2} a_{l} \mu\left(\ldots, \gamma_{1}, \gamma_{1}^{\prime}-1, r+l\right) \\
& +r(r-1) \alpha_{u} \sigma_{u}^{2} \mu(\ldots, r-2)
\end{aligned}
$$

with the notations $a_{0}=1, a_{1}=2 / \bar{U}$, and $a_{2}=1 / \bar{U}^{2}$. The set of these moment equations is expressed in closed form so that $\mu\left(\alpha_{1}, \alpha_{1}^{\prime}, \alpha_{2}, \alpha_{2}^{\prime}, \alpha_{3}, \alpha_{3}^{\prime}, r\right)$ can be exactly calculated. We obtain the moments of $Y_{k, x}(t)$ by setting $E\left[Y_{1, x}^{\alpha_{1}}(t) Y_{2, x}^{\beta_{1}}(t) Y_{3, x}^{\gamma_{1}}(t)\right]=\mu\left(\alpha_{1}, 0, \beta_{1}, 0, \gamma_{1}, 0,0\right)$.

Denote by $\mu_{i}(n)$ the $n^{t h}$ marginal moment $E\left[X_{i}^{n}(t)\right]$. We approximate the marginal distribution of $X_{i}(t)$ by $\hat{F}_{i}(x)=\sum_{j=1}^{N} p_{i j} \hat{F}_{i j}(x)$, where $\left\{\hat{F}_{i j}(x)\right\}$ are the preselected distributions with mean $\mu_{i}(1)$ and variance $\mu_{i}(2)-\mu_{i}(1)^{2},\left\{p_{i j} \geq 0\right\}$ are the parameters which minimize the error $\sum_{n=1}^{4}\left[\mu_{i}(n)-\hat{\mu}_{i}(n)\right]^{2}$ under the constraint $\sum_{j=1}^{N} p_{i j}=1$, in which $\left\{\hat{\mu}_{i}(n), n=1, \ldots, 4\right\}$ are the marginal moments of 
$\hat{F}_{i}(x)$. Note that $\hat{F}_{i}(x)$ are non-Gaussian since the along-wind forces are nonGaussian.

\subsection{Response translation models}

In the previous sections, we have shown that (1) structural responses are nonGaussian, and (2) the marginal distributions and the second-moment properties of the responses can be estimated. In order to model these responses, we use so-called translation models that constitute one-to-one mappings between Gaussian processes and non-Gaussian processes which have the target marginal distributions and similar second-moment properties. The components $X_{T, i}(t)$ of the translation model $\mathbf{X}_{T}(t)$ are defined by

$$
X_{T, i}(t)=\hat{F}_{i}^{-1}\left\{\Phi\left[Z_{i}(t)\right]\right\},
$$

where $\Phi$ denotes standard Gaussian distribution, and $\left\{Z_{i}(t)\right\}$ are the components of $\mathbf{Z}(t)$ which is a Gaussian vector process with zero mean, unit variance and spectral density $S_{\mathbf{Z}}(\nu) \geq 0$ that minimizes the difference between the spectral density of $\mathbf{X}_{T}(t), S_{\mathbf{x}_{T}}(\nu)$, and $S_{\mathbf{X}}(\nu)$. The properties of this translation model can be found in, e.g., [2, Section 3.1.1]. Based on the observation that the discrepancy between these two spectral densities is generally small irrespective of the choice of $S_{\mathbf{Z}}(\nu)$, we use for $S_{\mathbf{Z}}(\nu)$ a normalized version of $S_{\mathbf{X}}(\nu)$. The probability law of the translation model $\mathbf{X}_{T}(t)$ is fully characterized. 


\subsection{Mean outcrossing rates}

Suppose the system in Eq. 4.1 is safe if its responses approximated by $\mathbf{X}_{T}(t)$ do not leave the rectangular safe set $D_{x}=\times_{i=1}^{3}\left[-\xi x_{i}, \xi x_{i}\right]$, where $x_{i}>0$ is constant and $\xi>0$ is the scale factor controlling the size of the safe set. The average number crossings of $\mathbf{X}_{T}(t)$ out of $D_{x}$ per unit of time, referred to as mean $D_{x}$-outcrossing rate, are of interest. Denote the image of the safe set $D_{x}$ in the Gaussian space by $D_{z}=\times_{i=1}^{3}\left[z_{i}^{-}, z_{i}^{+}\right]$, where $z_{i}^{+}=\Phi^{-1}\left[\hat{F}_{i}\left(\xi x_{i}\right)\right]$ and $z_{i}^{-}=\Phi^{-1}\left[\hat{F}_{i}\left(-\xi x_{i}\right)\right]$. Since $\mathbf{X}_{T}(t)$ crosses safe set $D_{x}$ if and only if its mapped Gaussian image $\mathbf{Z}(t)$ crosses $D_{z}$, the mean $D_{x}$-outcrossing rate coincides with the mean $D_{z}$-outcrossing rate of $\mathbf{Z}(t)$, denoted by $\lambda_{D_{z}}$. Note that $\lambda_{D_{z}}$ for arbitrary safe set has the form [10, p. 300]

$$
\lambda_{D_{z}}=\int_{\partial D_{z}} u(\mathbf{z}) f(\mathbf{z}) d \sigma(\mathbf{z})
$$

where $\partial D_{z}$ denotes the boundaries of the safe set $D_{z}, \mathbf{z} \in \partial D_{z}, f(\mathbf{z})$ is the probability density function of $\mathbf{Z}(t), \sigma(\mathbf{z})$ is the surface area measured on $\partial D_{z}$, and

$$
u(\mathbf{z})=E\left[\dot{\mathbf{Z}}_{n}(t)+\mid \mathbf{Z}(t)=\mathbf{z}\right],
$$

where $\dot{\mathbf{Z}}_{n}(t)+$ denotes the positive projection of the velocity $\dot{\mathbf{Z}}(t)$ on the outer $\operatorname{normal} \mathbf{n}(\mathbf{z})$ to $\partial D_{z}$.

For a rectangular safe set $D_{z}$ with six planar boundaries, Eq. 4.12 yields

$$
\lambda_{D_{z}}=\sum_{i=1}^{3}\left[\lambda_{\partial D_{z_{i}^{+}}}+\lambda_{\partial D_{z_{i}^{-}}}\right]
$$

where $\lambda_{\partial D_{z_{i}^{+}}}$is the mean rate at which $Z_{i}(t)$ crosses boundary $\partial D_{z_{i}^{+}}$at distance $z_{i}^{+}$from the origin and with outer normal $\mathbf{n}_{i}^{+}$along with the axis corresponding to $Z_{i}(t)$, and $\lambda_{\partial D_{z_{i}^{-}}}$is defined similarly. $\lambda_{\partial D_{z_{i}^{+}}}$can be calculated by

$$
\lambda_{\partial D_{z_{i}^{+}}}=u_{i}\left(\mathbf{z}_{i}^{+}\right) P\left(\mathbf{Z}_{\neq i}(t) \in \partial D_{z_{i}^{+}} \mid Z_{i}(t)=z_{i}^{+}\right) f\left(z_{i}^{+}\right),
$$


where $\mathbf{Z}_{\neq i}(t)$ denotes $\mathbf{Z}(t)$ excluding $Z_{i}(t)$, and $u_{i}\left(\mathbf{z}_{i}^{+}\right)=E\left[\dot{Z}_{i}(t)+\mid \mathbf{Z}(t)=\mathbf{z}_{i}^{+}\right]$ is the corresponding $u(\mathbf{z})$ value on the boundary $\partial D_{z_{i}^{+}}$, in which $\dot{Z}_{i}(t)$ denotes the $i^{\text {th }}$ component of $\dot{\mathbf{Z}}(t)$ and $\mathbf{z}_{i}^{+} \in \partial D_{z_{i}^{+}}$. Note that $\dot{Z}_{i}(t)$ is independent of $\mathbf{Z}(t)$ under some conditions (See Appendix 2), and it is a Gaussian variable with mean zero and variance $\Gamma_{\dot{Z}_{i} \dot{Z}_{i}}$, where $\Gamma_{\dot{Z}_{i} \dot{Z}_{i}}=\int_{0}^{\infty} \nu^{2} S_{Z_{i} Z_{i}}(\nu) d \nu$ and $S_{Z_{i} Z_{i}}(\nu)$ is the spectral density of $Z_{i}(t)$. Accordingly, $u_{i}\left(\mathbf{z}_{i}^{+}\right)$becomes a constant $u_{i}^{+}$, i.e., $u_{i}^{+}=\left(\Gamma_{\dot{Z}_{i} \dot{Z}_{i}} / 2 \pi\right)^{1 / 2}$. The same procedure can be used to calculate $\lambda_{\partial D_{z_{i}^{-}}}$with threshold value $z_{i}^{-}$.

However, Eq. 4.15 may be computationally demanding because of the probability $P\left(\mathbf{Z}_{\neq i}(t) \in \partial D_{z_{i}^{+}} \mid Z_{i}(t)=z_{i}^{+}\right)$. We propose to estimate this term from samples of $\mathbf{Z}(t)$ for relatively small $z_{i}^{+}$and set $P\left(\mathbf{Z}_{\neq i}(t) \in \partial D_{z_{i}^{+}} \mid Z_{i}(t)=z_{i}^{+}\right) \simeq 1$ for relatively large values of $z_{i}^{+}$. Then, $\lambda_{\partial D_{z_{i}^{+}}} \simeq u_{i}^{+} f\left(z_{i}^{+}\right)$for relatively large $z_{i}^{+}$.

Fig. 4.2 shows the mean $D_{x}$-outcrossing rate of $\mathbf{X}(t)$. The black solid line is estimated from the response samples which are obtained from the Monte Carlo simulation based on samples of the empirical model, the red dash line is calculated from Eq. 4.14, and the blue dash line is the proposed approximation of Eq. 4.14. They are almost indistinguishable at the figure scale in the tail region.
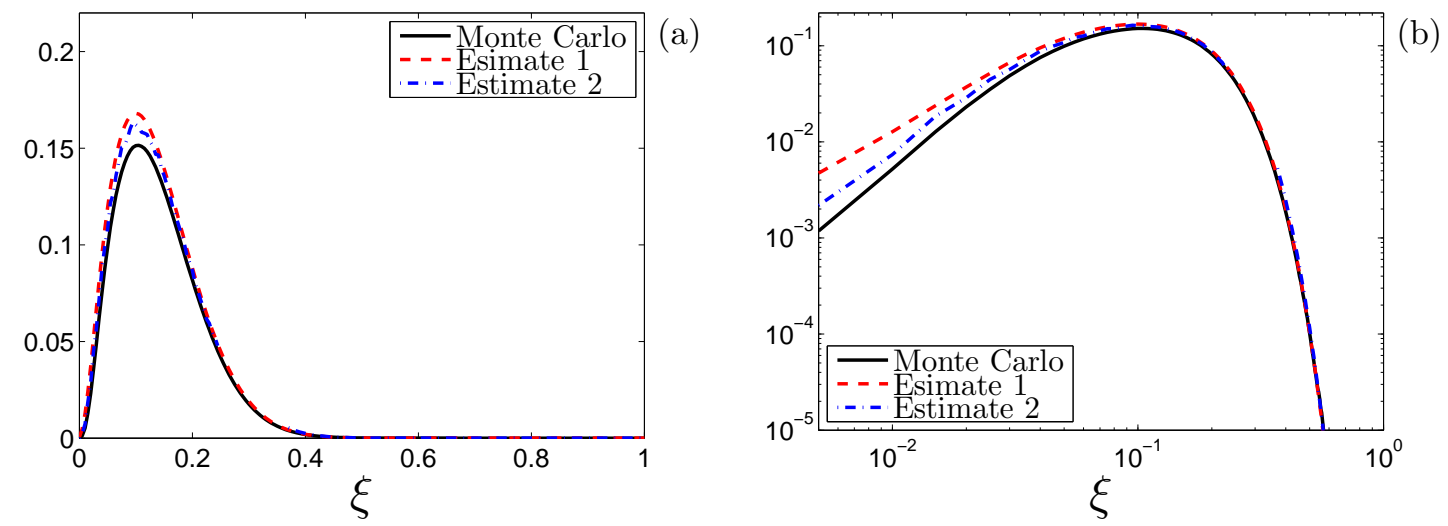

Figure 4.2: Mean $D_{x}$-outcrossing rates: linear (a) and logarithmic (b) scales 


\section{CHAPTER 5 CONCLUSIONS}

Probabilistic models have been constructed for wind loads based on (1) the existing wind-load model proposed in [7], and (2) the empirical or mathematical models for the second-moment properties of turbulence fluctuations and wake excitations. The empirical model follows from the experimental observations and knowledge of the physics, while the mathematical model represents the turbulence fluctuations and wake excitations by the filtered Gaussian processes with similar second-moment properties as the empirical model. We have shown that both models agree with the experimental observations.

The mathematical model provides an efficient method to estimate the response properties of linear systems relative to the classical Monte Carlo simulation. The responses are modeled by the translation processes calibrated to the second-moment properties and the marginal distributions of the responses. Linear random vibration theory is applied to find the target second-moment properties, and the marginal distributions match the exact marginal moments which are obtained by the moment equations developed from the Itô's formula. Then, response properties are approximated by the corresponding properties of the resulting translation processes. For example, crossing theory for translation processes is used to assess the structural reliability. The accuracy of this approximation is satisfactory as shown in the numerical example. 


\section{PROPERTY FROM FLOW SYMMETRY}

We prove that $E[\tilde{u}(t) \tilde{v}(t)]=0$ if the flow geometry is symmetric about the $x_{1}-x_{3}$ plane. Denote by $f\left(V_{1}, V_{2} ; \mathbf{x}, t\right)=\partial^{2} F\left(V_{1}, V_{2} ; \mathbf{x}, t\right) / \partial V_{1} \partial V_{2}$ the probability density function of wind-velocity vector $[u(\mathbf{x}, t), v(\mathbf{x}, t)]^{T}$, in which $\mathbf{x}=\left[x_{1}, x_{2}, x_{3}\right]$, and $F\left(V_{1}, V_{2} ; \mathbf{x}, t\right)=P\left(u(\mathbf{x}, t) \leq V_{1}, v(\mathbf{x}, t) \leq V_{2}\right)$. Because of the flow symmetry, the statistics is invariant under the reflection of the $x_{2}$ coordinate axis so that we have

$$
f\left(V_{1}, V_{2} ;\left[x_{1}, x_{2}, x_{3}\right], t\right)=f\left(V_{1},-V_{2} ;\left[x_{1},-x_{2}, x_{3}\right], t\right) .
$$

Then,

$$
\begin{aligned}
E\left[v\left(\left[0,0, H_{3}\right], t\right)\right] & =\int_{-\infty}^{\infty} \int_{-\infty}^{\infty} V_{2} f\left(V_{1}, V_{2} ;\left[0,0, H_{3}\right], t\right) d V_{1} d V_{2} \\
& =\int_{-\infty}^{\infty} \int_{-\infty}^{\infty}\left(-V_{2}\right) f\left(V_{1},-V_{2} ;\left[0,0, H_{3}\right], t\right) d V_{1} d V_{2}=-E\left[v\left(\left[0,0, H_{3}\right], t\right)\right]=0
\end{aligned}
$$

and

$$
\begin{aligned}
E[\tilde{u}(t) \tilde{v}(t)] & =E\left[u\left(\left[0,0, H_{3}\right], t\right) \tilde{v}\left(\left[0,0, H_{3}\right], t\right)\right]=E\left[u\left(\left[0,0, H_{3}\right], t\right) v\left(\left[0,0, H_{3}\right], t\right)\right] \\
& =\int_{-\infty}^{\infty} \int_{-\infty}^{\infty} V_{1} V_{2} f\left(V_{1}, V_{2} ;\left[0,0, H_{3}\right], t\right) d V_{1} d V_{2} \\
& =\int_{-\infty}^{\infty} \int_{-\infty}^{\infty}\left(-V_{1} V_{2}\right) f\left(V_{1},-V_{2} ;\left[0,0, H_{3}\right], t\right) d V_{1} d V_{2} \\
& =-E\left[u\left(\left[0,0, H_{3}\right], t\right) v\left(\left[0,0, H_{3}\right], t\right)\right]=-E\left[\tilde{u}\left(\left[0,0, H_{3}\right], t\right) \tilde{v}\left(\left[0,0, H_{3}\right], t\right)\right]=0 .
\end{aligned}
$$

Hence, we obtain the desired property. 


\section{APPENDIX B}

\section{PROOF OF INDEPENDENCE}

We prove that $\dot{Z}_{i}(t)$ is independent of $\mathbf{Z}(t)$ under some conditions. Without loss of generality, set $i=1$.

The proof has two steps. First, let $\varphi\left(\nu_{1}, \nu_{2}, \nu_{3}, \nu\right)=E\left[\exp \left\{\left(\sum_{j=1}^{3} \nu_{j} Z_{j}(t)+\right.\right.\right.$ $\left.\left.\left.\nu Y_{n}(t)\right) i\right\}\right]$ be the characteristic function of vector $\left[Z_{1}(t), Z_{2}(t), Z_{3}(t), Y_{n}(t)\right]$, where $Y_{n}(t)=\left(Z_{1}\left(t+h_{n}\right)-Z_{1}(t)\right) / h_{n}$. we show that $\lim _{h_{n} \rightarrow 0} \varphi\left(\nu_{1}, \nu_{2}, \nu_{3}, \nu\right)=$ $E\left[\exp \left\{\left(\sum_{j=1}^{3} \nu_{j} Z_{j}(t)+\nu \dot{Z}_{1}(t)\right) i\right\}\right]$ which is the characteristic function of vector $\left[Z_{1}(t), Z_{2}(t), Z_{3}(t), \dot{Z}_{1}(t)\right]$. Under the assumption that $Z_{1}(t)$ is mean square differentiable, $Y_{n}(t) \longrightarrow \dot{Z}_{1}(t)$ in $L_{2}$ so that $Y_{n}(t) \stackrel{p}{\longrightarrow} \dot{Z}_{1}(t)$ by Chebyshev's inequality [3, p. 143]. Set $\tilde{Y}_{n}(t)=\sum_{j=1}^{3} \nu_{j} Z_{j}(t)+\nu Y_{n}(t)$ and $\tilde{Z}(t)=\sum_{j=1}^{3} \nu_{j} Z_{j}(t)+\nu \dot{Z}_{1}(t)$. We have $\tilde{Y}_{n}(t) \stackrel{p}{\longrightarrow} \tilde{Z}(t)$, then each subsequence $\tilde{Y}_{n_{k}}(t)$ of $\tilde{Y}_{n}(t)$ contains a subsequence $\tilde{Y}_{n_{k_{q}}}(t)$ such that $\tilde{Y}_{n_{k_{q}}}(t) \stackrel{\text { a.s. }}{\longrightarrow} \tilde{Z}(t)[3$, p. 72]. Hence, we obtain the desired results by the continuity of characteristic function and Bounded Convergence Theorem.

Second, we derive the expression for $\lim _{h_{n} \rightarrow 0} \varphi\left(\nu_{1}, \nu_{2}, \nu_{3}, \nu\right)$. Note that $\lim _{h_{n} \rightarrow 0} \varphi\left(\nu_{1}, \nu_{2}, \nu_{3}, \nu\right)$ can be expressed by

$\lim _{h_{n} \rightarrow 0} \varphi\left(\nu_{1}, \nu_{2}, \nu_{3}, \nu\right)=E\left[\exp \left(\left\{\left(\nu_{1}-\frac{\nu}{h_{n}}\right) Z_{1}(t)+\frac{\nu}{h_{n}} Z_{1}\left(t+h_{n}\right)+\nu_{2} Z_{2}(t)+\nu_{3} Z_{3}(t)\right\} i\right)\right]$.

Since $Z_{1}(t), Z_{2}(t)$, and $Z_{3}(t)$ are standard Gaussian processes, Eq. B.1 yields

$$
\begin{aligned}
\lim _{h_{n} \rightarrow 0} \varphi\left(\nu_{1}, \nu_{2}, \nu_{3}, \nu\right) & =\lim _{h_{n} \rightarrow 0} \exp \left[-\frac{1}{2}\left\{\left(2-2 \rho_{11}\left(h_{n}\right)\right) \frac{\nu^{2}}{h_{n}^{2}}+\left(2 \rho_{11}\left(h_{n}\right)-2\right) \frac{\nu \nu_{1}}{h_{n}}\right.\right. \\
& \left.\left.+\left(2 \rho_{12}\left(h_{n}\right)-2 \rho_{12}(0)\right) \frac{\nu \nu_{2}}{h_{n}}+\left(2 \rho_{13}\left(h_{n}\right)-2 \rho_{13}(0)\right) \frac{\nu \nu_{3}}{h_{n}}+\boldsymbol{\nu}^{T} \Sigma \boldsymbol{\nu}\right\}\right],
\end{aligned}
$$


where $\rho_{p q}\left(h_{n}\right)=E\left[Z_{p}\left(t+h_{n}\right) Z_{q}(t)\right], p, q=1,2,3, \quad \nu=\left[\nu_{1}, \nu_{2}, \nu_{3}\right]^{T}$, and $\Sigma$ is a $3 \times 3$ matrix whose first, second, and third columns are $\left[1, \rho_{12}(0), \rho_{13}(0)\right]^{T},\left[\rho_{12}(0), 1, \rho_{23}(0)\right]^{T}$, and $\left[\rho_{13}(0), \rho_{23}(0), 1\right]^{T}$, respectively. Since $Z_{1}(t)$ is stationary and mean square differentiable, $-\partial^{2} \rho_{11}\left(h_{n}\right) /\left.\partial h_{n}^{2}\right|_{h_{n}=0}$ exists and $\partial \rho_{11}\left(h_{n}\right) /\left.\partial h_{n}\right|_{h_{n}=0}=0$. Under the hypotheses that $\rho_{12}\left(h_{n}\right)=\rho_{12}\left(-h_{n}\right)$ and $\rho_{13}\left(h_{n}\right)=\rho_{13}\left(-h_{n}\right), \partial \rho_{12}\left(h_{n}\right) /\left.\partial h_{n}\right|_{h_{n}=0}=0$ and $\partial \rho_{13}\left(h_{n}\right) /\left.\partial h_{n}\right|_{h_{n}=0}=0$. So we have

$$
\lim _{h_{n} \rightarrow 0} \varphi\left(\nu_{1}, \nu_{2}, \nu_{3}, \nu\right)=\exp \left[-\frac{\nu^{2}}{2}\left\{-\left.\frac{\partial^{2} \rho_{11}\left(h_{n}\right)}{\partial h_{n}^{2}}\right|_{h_{n}=0}\right\}\right] \exp \left[-\frac{1}{2} \boldsymbol{\nu}^{T} \Sigma \boldsymbol{\nu}\right]
$$

in which the first term on the right-hand side is the characteristic function of $\dot{Z}_{1}(t)$ and the second one is the characteristic function of $\mathbf{Z}(t)$. Hence, $\dot{Z}_{1}(t)$ is independent of $\mathbf{Z}(t)$. 


\section{BIBLIOGRAPHY}

[1] J. B. Barlow, W.H. Rae, and A. Pope. Low-Speed Wind Tunnel Testing. John Wiley \& Sons, New York, USA, third edition, 1999.

[2] M. Grigoriu. Applied Non-Gaussian Processes: Examples, Theory, Simulation, Linear Random Vibration, and MATLAB Solutions. PTR Prentice Hall, Englewood Cliffs, NJ, USA, 1995.

[3] M. Grigoriu. Stochastic Calculus: Applications in Science and Engineering. Birkhäuser, Boston, MA, USA, 2002.

[4] M. Grigoriu and S. T. Ariaratnam. Response of linear systems to polynomials of gaussian processes. Journal of Applied Mechanics, 55(4):905-910, 1988.

[5] M. Grigoriu and R. V. Field. A method for analysis of linear dynamic systems driven by stationary non-gaussian noise with applications to turbulence-induced random vibration. Applied Mathematical Modelling, 38(1):336-354, 2014.

[6] H. Kikuchi, Y. Tamura, H. Ueda, and K. Hibi. Dynamic wind pressures acting on a tall building model-proper orthogonal decomposition. Journal of Wind Engineering and Industrial Aerodynamics, 69-71:631-646, 1997.

[7] G. Solari. Gust-excited vibrations. Wind-excited Vibrations of Structures, 335:195-291, 1994.

[8] G. Solari and G. Piccardo. Probabilistic 3-d turbulence modeling for gust buffeting of structures. Probabilistic Engineering Mechanics, 16(1):73-86, 2001.

[9] G. Solari and F. Tubino. Dynamic approach to the wind loading of structures: alongwind, crosswind and torsional response. Wind Effects on Buildings and Design of Wind-sensitive Structures, 493:137-166, 2007.

[10] T. T. Soong and M. Grigoriu. Random Vibration of Mechanical and Structural Systems. Prentice Hall, Englewood Cliffs, NJ, USA, 1993.

[11] B. J. Vickery and A. W. Clark. Lift or across-wind response to tapered stacks. Journal of the Structural Division, 98(1):1-20, 1972. 